\title{
Characterizing graphs of maximum matching width at most 2
}

\author{
Jeong, Jisu ; Ok, Seongmin; Suh, Geewon
}

Published in:

Discrete Applied Mathematics

Link to article, DOI:

10.1016/j.dam.2017.07.028

Publication date:

2017

Document Version

Version created as part of publication process; publisher's layout; not normally made publicly available

Link back to DTU Orbit

Citation (APA):

Jeong, J., Ok, S., \& Suh, G. (2017). Characterizing graphs of maximum matching width at most 2. Discrete Applied Mathematics, 248, 102-113. https://doi.org/10.1016/j.dam.2017.07.028

\section{General rights}

Copyright and moral rights for the publications made accessible in the public portal are retained by the authors and/or other copyright owners and it is a condition of accessing publications that users recognise and abide by the legal requirements associated with these rights.

- Users may download and print one copy of any publication from the public portal for the purpose of private study or research.

- You may not further distribute the material or use it for any profit-making activity or commercial gain

- You may freely distribute the URL identifying the publication in the public portal

If you believe that this document breaches copyright please contact us providing details, and we will remove access to the work immediately and investigate your claim. 


\title{
Characterizing graphs of maximum matching width at most 2
}

\author{
Jisu Jeong ${ }^{\mathrm{a}, *}$, Seongmin Ok ${ }^{\mathrm{b}}$, Geewon Suh ${ }^{\mathrm{c}}$ \\ a Department of Mathematical Sciences, Korea Advanced Institute of Science and Technology, Daejeon, Republic of Korea \\ b School of Computational Sciences, Korea Institute for Advanced Study, Seoul, Republic of Korea \\ c School of Electrical Engineering, Korea Advanced Institute of Science and Technology, Daejeon, Republic of Korea
}

\section{A R T I C L E I N F O}

\section{Article history:}

Received 3 June 2016

Received in revised form 13 June 2017

Accepted 23 July 2017

Available online $\mathrm{xxxx}$

\section{Keywords:}

Maximum matching width

Minor obstructions

Grid

\begin{abstract}
A B S T R A C T
The maximum matching width is a width-parameter that is defined on a branchdecomposition over the vertex set of a graph. The size of a maximum matching in the bipartite graph is used as a cut-function. In this paper, we characterize the graphs of maximum matching width at most 2 using the minor obstruction set. Also, we compute the exact value of the maximum matching width of a grid.
\end{abstract}

(c) 2017 Elsevier B.V. All rights reserved.

\section{Introduction}

Treewidth and branchwidth are well-known width-parameters of graphs used in structural graph theory and theoretical computer science. Based on Courcelle's theorem [4], which states that every property on graphs definable in monadic secondorder logic can be decided in linear time on a class of graphs with bounded treewidth, many NP-hard problems have been shown to be solvable in polynomial time by dynamic programming when the input has bounded treewidth or branchwidth.

Vatshelle [20] introduced a new graph width-parameter, called the maximum matching width (mm-width in short), that uses the size of a maximum matching as a cut-function in its branch-decomposition of the vertex set of a graph. Maximum matching width is related to treewidth and branchwidth as shown by the inequality $\operatorname{mmw}(G) \leq \max (\operatorname{brw}(G), 1) \leq$ $\operatorname{tw}(G)+1 \leq 3 \mathrm{mmw}(G)$ for every graph $G[20]$ where $\operatorname{mmw}(G), \operatorname{tw}(G)$, and $\operatorname{brw}(G)$ are the maximum matching width, the treewidth, and the branchwidth of $G$ respectively. This implies that bounding the treewidth or branchwidth is qualitatively equivalent to bounding the maximum matching width. Maximum matching width gives a more efficient algorithm for some problems. For a given branch-decomposition of a graph $G$ of maximum matching width $k$, we can solve the Minimum Dominating Set Problem in time $0^{*}\left(8^{k}\right)$ [8], which gives a better runtime than $O^{*}\left(3^{\mathrm{tw}(G)}\right)$-time algorithm in [19] when $\operatorname{tw}(G)>\left(\log _{3} 8\right) k$. Note that the Minimum Dominating Set Problem cannot be solved in time $0^{*}\left((3-\varepsilon)^{\operatorname{tw}(G)}\right)$ for every $\varepsilon>0$ unless the Strong Exponential Time Hypothesis fails [10].

The Robertson-Seymour theorem [13] states that every minor-closed class of graphs has a finite minor obstruction set. In the other words, a graph $G$ is in the class if and only if $G$ has no minor isomorphic to a graph in the obstruction set. Much work has been done to identify the minor obstruction set for various graph classes, especially for graphs of bounded width-parameters [2,5,9].

Let $K_{n}, C_{n}$, and $P_{n}$ be the complete graph, the cycle graph, and the path graph on $n$ vertices, respectively. The graphs $K_{3}$ and $K_{4}$ are the unique minor obstruction for the graphs of treewidth at most 1 and 2 [21], respectively. The minor obstruction set for the class of graphs having treewidth at most 3 is $\left\{K_{5}, K_{2,2,2}, K_{2} \times C_{5}, M_{8}\right\}$ where $K_{2} \times C_{5}$ is the Cartesian product of $K_{2}$ and $C_{5}$, and $M_{8}$ is the Wagner graph, also called the Möbius ladder with eight vertices $[1,16]$.

Robertson and Seymour [12] gave a characterization for the classes of graphs of branchwidth at most 1 and at most 2. The graphs $K_{3}$ and $P_{4}$ are forbidden minors for the graphs of branchwidth at most 1 . For the class of graphs of branchwidth

\footnotetext{
* Corresponding author.

E-mail addresses: jjisu@kaist.ac.kr (J. Jeong), seong@kias.re.kr (S. Ok), gwsuh91@kaist.ac.kr (G. Suh).
} 
at most 2, its minor obstruction is the same as treewidth, which is $K_{4}$. The graphs of branchwidth at most 3 have four minor obstructions; $\left\{K_{5}, K_{2,2,2}, K_{2} \times C_{4}, M_{8}\right\}$ [3].

One of the main results of this paper is to find the minor obstruction set for the class of graphs of mm-width at most 2. Note that the class of graphs with bounded mm-width is closed under taking minor, as shown in Corollary 2.3. Our main result is the following.

Theorem 3.17. Let $\mathcal{O}=\mathcal{O}_{3} \cup \mathcal{O}_{4} \cup \mathcal{O}_{5} \cup \mathcal{O}_{6}$ be the set of 42 graphs in Figs. 1, 5-7. A graph $G$ has mm-width at most 2 if and only if $G$ has no minor isomorphic to a graph in $\mathcal{O}$.

The exact value of some width-parameters for grid graphs is well known. For an integer $k \geq 1$, the branchwidth and treewidth of the $k \times k$-grid are $k$ [12,17], and the rank-width of the $k \times k$-grid is $k-1$ [7]. From the inequality $\operatorname{rw}(G) \leq \operatorname{mmw}(G) \leq \max (\operatorname{brw}(G), 1)$ [20], the mm-width of the $k \times k$-grid is either $k-1$ or $k$. Our second result is that the latter is the right answer when $k \geq 2$.

Theorem 4.7. The $k \times k$-grid has mm-width $k$ for $k \geq 2$.

Section 2 lists some of the definitions, including a tangle, and provides preliminaries for the maximum matching width. In Section 3 we identify the minor obstruction set for graphs with mm-width at most 2 . Section 4 gives the result for the precise mm-width of the square grids.

\section{Preliminaries}

Every graph $G=(V, E)$ in this paper is finite and simple. For a set $X \subseteq V(G) \cup E(G)$, we write $G \backslash X$ to denote the graph obtained from $G$ by deleting all vertices and edges in $X$. If $X \subseteq E(G)$, we write $G / X$ to denote the graph obtained from $G$ by contracting the edges in $X$. If $X=\{x\}$, then we write $G \backslash x$ and $G / x$ instead of $G \backslash X$ and $G / X$, respectively. If a subgraph $G^{\prime}$ of $G$ with $V\left(G^{\prime}\right)=X$ contains all the edges of $G$ whose both ends are in $X$, then we call $G^{\prime}$ induced by $X$ and write $G^{\prime}:=G[X]$. For a graph $G$ and disjoint subsets $X, Y \subseteq V(G)$, let $E_{G}[X, Y]$ (or $E[X, Y]$ ) denote the set of all edges $e=u v$ where $u$ is in $X$ and $v$ is in $Y$, and let $G[X, Y]=G(X \cup Y, E[X, Y])$. A graph $G$ is $k$-connected if $|V(G)| \geq k$ and $G \backslash X$ is connected for every $X \subset V(G)$ with $|X|<k$. A bridge is an edge $e$ such that $G \backslash e$ has more components than $G$. A block is either a bridge as a subgraph or a maximal 2-connected subgraph.

We say that a tree is ternary if all vertices have degree 1 or 3 . A branch-decomposition of a finite set $X$ is a pair $(T, \mathcal{L})$ of a ternary tree $T$ together with a bijection $\mathcal{L}$ from the leaves of $T$ to $X$. Note that an edge $a b$ of $T$ partitions the leaves of $T$ into two parts, say $A$ and $B$. We say an edge $e$ induces the partition $(A, B)$. A function $f: 2^{X} \rightarrow \mathbb{Z}$ is symmetric if $f(A)=f(X \backslash A)$ for all $A \subseteq X$, and the function $f$ is submodular if $f(A)+f(B) \geq f(A \cup B)+f(A \cap B)$ for all $A, B \subseteq X$. For each edge $e$ of $T$, and a symmetric, submodular function $f$, the $f$-value of $e$ is equal to $f(A)=f(B)$ where $(A, B)$ is the partition induced by $e$. The $f$-width of a branch-decomposition $(T, \mathcal{L})$ is the maximum $f$-value of an edge of $T$, and the $f$-width of $X$ is the minimum value of the $f$-width over all possible branch-decompositions of $X$. This notion of $f$-width provides a link between several width parameters.

For $A \subseteq E(G)$, let $b r: 2^{E(G)} \rightarrow \mathbb{Z}$ be the function so that $\operatorname{br}(A)$ is the number of vertices that are incident to both an edge in $A$ and an edge in $E(G) \backslash A$. The branchwidth of $G$, denoted by brw $(G)$, is the br-width of $E(G)$.

For $A \subseteq V(G)$, let $r: 2^{V(G)} \rightarrow \mathbb{Z}$ be the function such that $r(A)$ is the rank of the adjacency matrix between $A$ and $V(G) \backslash A$ over $\mathbb{F}_{2}$. The rank-width of $G$, denoted by $\operatorname{rw}(G)$, is the $r$-width of $V(G)$.

Let $\mathrm{mm}_{G}: 2^{V(G)} \rightarrow \mathbb{Z}$ be the function such that $\mathrm{mm}_{G}(A)$ is the size of a maximum matching in $G[A, V(G) \backslash A]$. Note that the function $\mathrm{mm}_{G}$ is symmetric and submodular [15]. We use $\mathrm{mm}$ instead of $\mathrm{mm}_{G}$ if the host graph $G$ is clear from the context. The maximum matching width of $G$, denoted by $\operatorname{mmw}(G)$, is the mm-width of $V(G)$.

A graph $H$ is a minor of a graph $G$ if $H$ can be constructed from $G$ by deleting edges, deleting vertices, and contracting edges. We call a graph $G$ minor-minimal with respect to a property $\mathcal{P}$ if $G$ has $\mathcal{P}$ but no proper minor of $G$ has $\mathcal{P}$. A graph $G$ is a forbidden minor of a graph class $\mathcal{C}$ if $H \notin \mathcal{C}$ whenever $H$ has a minor isomorphic to $G$. Robertson and Seymour [13] state that the collection of minor-minimal graphs outside a minor-closed graph class is finite. The collection is called the minor obstruction set.

A graph is chordal if every induced cycle in the graph has length 3. A chordalization of a graph $G$ is a chordal graph $H$ such that $V(H)=V(G)$ and $E(G) \subseteq E(H)$. An intersection graph $G$ over a family $\left\{A_{i}\right\}$ of sets is the graph with $V(G)=\left\{A_{i}\right\}$ and $E(G)=\left\{A_{i} A_{j}: A_{i} \cap A_{j} \neq \emptyset\right\}$. Remark that a graph is chordal if and only if it is the intersection graph of the edge sets of subtrees of a tree [6].

\subsection{Maximum matching width}

Jeong, Sæther, and Telle [8] gave a new characterization of graphs of mm-width at most $k$ as an intersection graph by the following theorem. A tree is called nontrivial if it has at least one edge and a tree is subcubic if all vertices have degree at most 3.

Theorem $2.1([8])$. The maximum matching width of a graph $G$ is at most $k$ if and only if there exist a subcubic tree $T$ and a set $\left\{T_{x}\right\}_{x \in V(G)}$ of nontrivial subtrees of $T$ such that 
(1) if $u v \in E(G)$, then the subtrees $T_{u}$ and $T_{v}$ have at least one vertex of $T$ in common,

(2) for each edge e of $T$ there are at most $k$ subtrees in $\left\{T_{x}\right\}_{x \in V(G)}$ containing $e$.

Note that we can replace a subcubic tree $T$ to a ternary tree $T$ in Theorem 2.1 because we can add an edge to each degree- 2 vertex of $T$.

A tree-representation of $G$ having width at most $k$ is a pair $\left(T,\left\{T_{x}\right\}_{x \in V(G)}\right)$ where $T$ is a ternary tree and a set $\left\{T_{x}\right\}_{x \in V(G)}$ of nontrivial subtrees satisfying the properties (1) and (2). Theorem 2.1 says that a graph $G$ has a tree-representation of width at most $k$ if and only if $\operatorname{mmw}(G) \leq k$.

For a tree-representation $\left(T,\left\{T_{x}\right\}\right)_{x \in V(G)}$ of $G$, the intersection graph $G_{T}$ of the family $\left\{T_{x}\right\}_{x \in V(G)}$ is chordal and $G$ is a subgraph of $G_{T}$. Since $G$ and $G_{T}$ have the same tree-representation $\left(T,\left\{T_{x}\right\}\right)_{x \in V(G)}$, every graph has a chordalization with the same mmwidth.

It is easy to check that, for a graph $G$ and its vertex or edge $x$,

$$
\operatorname{mmw}(G \backslash x) \leq \operatorname{mmw}(G) .
$$

The same holds for contracting an edge.

Lemma 2.2. Let $G$ be a graph. For every edge $u v$ of $G, \operatorname{mmw}(G / u v) \leq \operatorname{mmw}(G)$.

Proof. Let $\left(T,\left\{T_{x}\right\}_{x \in V(G)}\right)$ be a tree-representation of $G$ having width $\operatorname{mmw}(G)$. Let $T_{u v}$ be the subtree of $T$ with vertex set $V\left(T_{u}\right) \cup V\left(T_{v}\right)$ and edge set $E\left(T_{u}\right) \cup E\left(T_{v}\right)$. Then $\left(T,\left\{T_{x}\right\}_{x \in V(G) \backslash\{u, v\}} \cup\left\{T_{u v}\right\}\right)$ is a tree-representation of $G / u v$ having width at most $\operatorname{mmw}(G)$. By Theorem 2.1, $\operatorname{mmw}(G / u v) \leq \operatorname{mmw}(G)$.

Corollary 2.3. Let $k$ be an integer. The set $M_{k}=\{G: \operatorname{mmw}(G) \leq k\}$ is closed under the minor operations.

By Corollary 2.3 and Robertson-Seymour theorem [13], $M_{k}$ has a finite minor obstruction set for each $k$. We can easily find the minor obstruction set when $k=1$.

Proposition 2.4 ([14]). A graph $G$ has mm-width at most 1 if and only if $G$ does not contain $C_{4}$ as a minor.

Proof. Suppose that $G$ contains $C_{4}$ as a minor. We can find four vertices $v_{1}, v_{2}, v_{3}, v_{4}$ of $G$ and four paths $P_{12}, P_{23}, P_{34}, P_{41}$ in $G$ such that each path $P_{i j}$ is a path from $v_{i}$ to $v_{j}$ and the four paths are pairwise internally vertex-disjoint. For every branchdecomposition $(T, \mathcal{L})$ of $V(G)$, there exists an edge $e$ in $T$ that induces a partition $(A, B)$ of $V(G)$ such that two vertices from $v_{1}, v_{2}, v_{3}, v_{4}$ are in $A$ and the other two are in $B$. Thus, there exist two vertex-disjoint paths from $A$ to $B$. This implies that the $\mathrm{mm}_{G}$-value of $e$ is at least 2 , and therefore $G$ has mm-width at least 2 .

Now let us suppose that $G$ does not contain $C_{4}$ as a minor. It is easy to see that every block of $G$ is either $K_{2}$ or $C_{3}$. The $\mathrm{mm}$-width of $G$ is the maximum value among the mm-widths of blocks of $G$. Since both $K_{2}$ and $C_{3}$ have mm-width $1, G$ has mm-width at most 1.

\subsection{Tangle}

Before proving our main theorems, we shall introduce the notion of tangle, which is useful in investigating the lower bounds of width-parameters.

Let $f$ be an integer-valued symmetric submodular function on the subsets of a finite set $X$. An $f$-tangle of order $k+1$ is a collection $\mathcal{T}$ of subsets of $X$ satisfying that

(T1) for all $S \subseteq X$, if $f(S) \leq k$, then one of $S$ and $X \backslash S$ is in $\mathcal{T}$,

(T2) if $S_{1}, S_{2}, S_{3} \in \mathcal{T}$, then $S_{1} \cup S_{2} \cup S_{3} \neq X$,

(T3) for each $x \in X, X \backslash\{x\} \notin \mathcal{T}$.

Robertson and Seymour [12] proved the following theorem. We use it in both Sections 3 and 4.

Theorem 2.5 ([12]). Let $f$ be an integer-valued symmetric submodular function on subsets of a finite set $X$. The $f$-width of $X$ is larger than $k$ if and only if there exists an $f$-tangle of order $k+1$.

The $k \times k$-grid, denoted by $G_{k}$, is the graph with vertex set $V\left(G_{k}\right)=\{(i, j): 1 \leq i, j \leq k\}$ and edge set $E\left(G_{k}\right)=\left\{(i, j)\left(i^{\prime}, j^{\prime}\right)\right.$ : $\left.\left|i-i^{\prime}\right|+\left|j-j^{\prime}\right|=1\right\}$. Using Theorem 2.5, we show that the $3 \times 3$-grid has mm-width 3 , as an example.

Lemma 2.6. The $3 \times 3$-grid $G_{3}$ has an mm-tangle of order 3 .

Proof. Let us consider $G_{3}$ to be a part of an integer grid in the real plane and let $\{(i, j): 1 \leq i, j \leq 3\}$ be the vertex set of $G_{3}$. Let $A$ be a set of all subsets of $V\left(G_{3}\right)$ with size at most 2 . Let $B=\{\{(1,1),(1,2),(2,1)\},\{(1,2),(1,3),(2,3)\},\{(2,3),(3,2),(3,3)\}$, $\{(2,1),(3,1),(3,2)\}\}$. We claim that $A \cup B$ is an mm-tangle of order 3 . It is trivial that (T3) holds. If $S_{1} \cup S_{2} \cup S_{3}=V\left(G_{3}\right)$, 
then the sets $S_{1}, S_{2}, S_{3}$ must be in $B$. However, no set in $B$ has $(2,2)$ and thus (T2) follows. Now we check (T1). Note that for every subset $S \subseteq V$ with $|S|=4$, we have $m m(S) \geq 3$. Since $A$ contains all subsets of size at most 2 , we need to consider subsets of $V\left(G_{3}\right)$ of size 3 . The elements in $B$ are the only subsets of size 3 having $\mathrm{mm}(S) \leq 2$. Hence (T1) holds too and $A \cup B$ is a mm-tangle of order 3.

By Lemma 2.6 and Theorem 2.5, the $3 \times 3$-grid has mm-width at least 3. It is easy to see that the $3 \times 3$-grid has mm-width at most 3 since it has 9 vertices and $K_{9}$ has a tree-representation of width 3 . Thus the $3 \times 3$-grid has mm-width 3. In this paper, we use a similar argument to verify that the graphs in the minor obstruction set for mm-width at most 2 have mm-width 3 . Note that the $3 \times 3$-grid is also in the minor obstruction set for the graphs of mm-width at most 2 . See Fig. 6(b).

\section{Minor obstruction set for maximum matching width at most 2}

Note that if $G$ is not 2-connected, then $\operatorname{mmw}(G)$ is the maximum of $\operatorname{mmw}(H)$ where $H$ is a maximal 2-connected subgraph of $G$. Thus the graphs in the minor obstruction set are 2-connected.

In Section 3.1 we identify the 3 -connected graphs that are minor-minimal with respect to mm-width $\geq 3$. And then we consider the minor-obstructions with 2-cuts in Section 3.2. We shall show that each 2-cut separates the graph into at most three components, where all but one component is small (a full characterization is given after Lemma 3.7). We show that the obstructions are obtained from a 3-connected graph with $\leq 6$ vertices by replacing some edges with small components mentioned above. What remains is to check all the candidates.

\subsection{3-connected graphs}

In this subsection, we give five 3-connected graphs that have mm-width 3 and whose proper minors have mm-width 2.

Lemma 3.1. For each ternary tree $T$, one can find an edge of $T$ whose removal divides the set of leaves into two subsets, each having at least $1 / 3$ of all the leaves.

Proof. Let $e=u v$ be an edge that induces a partition $(A, B)$ of the leaves where $u$ is on the side of $A$. Suppose that $A$ contains more than $2 / 3$ of the leaves. Then $u$ has degree 3 and the other two edges at $u$ induce leaf partitions, namely $\left(A_{1}, B_{1}\right)$ and $\left(A_{2}, B_{2}\right)$ where we assume $u$ to be on the side of $A_{1}$ and $A_{2}$ respectively. We choose the edge, say $e^{\prime}$, with larger $\left|A_{i}\right|$. If both $A_{1}$ and $A_{2}$ contain at most $2 / 3$ of the leaves then $e^{\prime}$ will be the edge we are after. Otherwise, we have a partition with smaller difference $\left|A_{i}\right|-\left|B_{i}\right|$ than $|A|-|B|$ and we iterate until we find a working edge.

By Lemma 3.1, a ternary tree with at least 7 leaves has an edge dividing the leaves into two sets such that both have size at least 3.

Lemma 3.2. If a graph $G$ is 3-connected and $G$ has at least 7 vertices, then $m m w(G) \geq 3$.

Proof. By the argument above, for every branch decomposition $(T, \mathcal{L})$ of $V(G)$, we can find an edge $e$ in $T$ inducing a partition $(A, B)$ with $|A|,|B| \geq 3$. Since $G$ is 3-connected, by Menger's theorem, $G$ has three vertex-disjoint paths between $A$ and $B$. These paths give a matching of size 3 in $G[A, B]$, which means that the $m_{G}$-value of $e$ is at least 3 . Thus, every branchdecomposition of $V(G)$ has $\mathrm{mm}_{G}$-width at least 3 .

It is easy to find a tree-representation of $K_{3 n}$ with width $n$. In particular, $K_{6}$ has mm-width 2 and hence every graph on 6 vertices has mm-width at most 2 . In other words, the forbidden minors for mm-width at most 2 have at least 7 vertices. We use Tutte's wheel theorem stated below. In the following statement we assume pairwise parallel edges occurring from contractions are all removed but one to keep the graph simple.

Theorem 3.3 (Tutte's Wheel Theorem [18]). If a graph $G$ is 3-connected, then $G$ has an edge e such that either G/e or $G \backslash e$ is 3-connected unless $G=K_{4}$.

Lemma 3.4. Let $\mathcal{O}_{3}$ be the set of the five graphs in Fig. 1. A 3-connected graph is minor-minimal with respect to maximum matching width at least 3 if and only if it is in $\mathrm{O}_{3}$.

Proof. By Tutte's wheel theorem, a 3-connected graph with at least 8 vertices has a proper 3-connected minor with at least 7 vertices, which has mm-width at least 3 by Lemma 3.2. Thus a minor-minimal 3-connected graph with respect to mm-width at least 2 has precisely 7 vertices. By [11], the five graphs in Fig. 1 are precisely the edge-minimal 3-connected graphs on 7 vertices, and hence it is enough to show that the proper minors of these graphs all have mm-width at most 2.

Observe that all edges of a graph in $\mathcal{O}_{3}$ are incident with a vertex of degree 3 . Thus by taking out the edge we have a graph on 7 vertices with at least one vertex of degree 2, say $v$. Starting from a tree-representation of $G \backslash v$ with width 2 , by rearranging the leaves if needed, we can easily add a vertex $v$ of degree 2 without increasing mm-width, so such a graph must have mm-width 2 . 

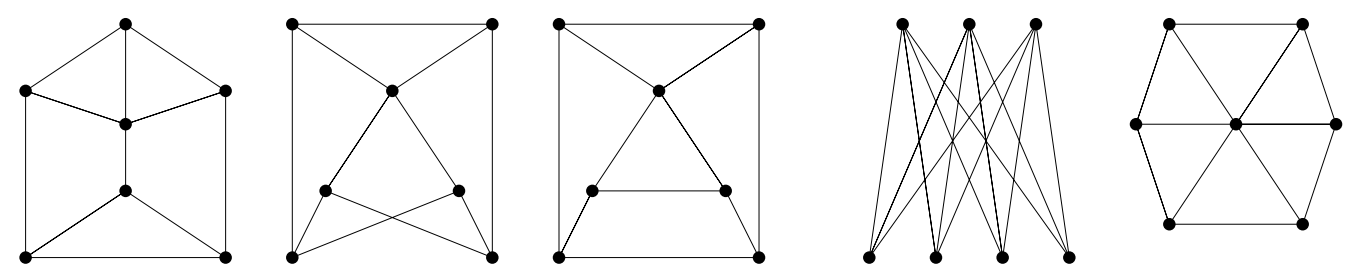

Fig. 1. The minor-minimal 3-connected graphs on 7 vertices.
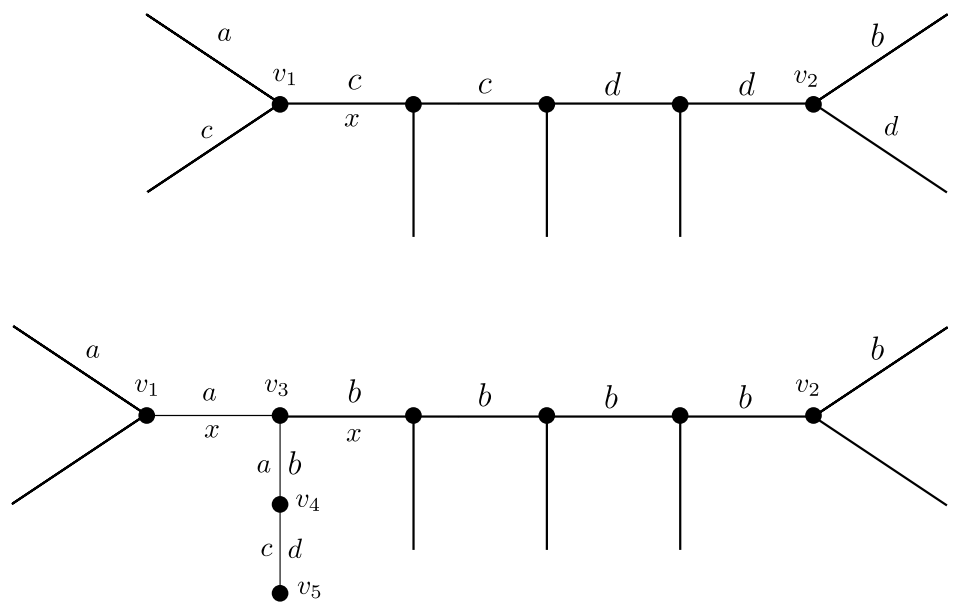

Fig. 2. Another tree-representation of the same graph where $T_{a}$ and $T_{b}$ share an edge.

\subsection{2-connected graphs}

Now we find 2-connected minor-minimal graphs with respect to mm-width 3 that are not 3-connected. Let $\mathcal{O}_{2}$ be the set of all graphs $G$ such that $G$ is not 3-connected and $G$ is minor-minimal with respect to mm-width at least 3 . Note that the graphs in $\mathrm{O}_{2}$ are 2-connected.

We say that a tree-representation of $G$ is good if there exist two vertices $a$ and $b$ such that the subtrees for $a$ and $b$ share an edge and the width of the tree-representation is 2 . A pair $(G,\{a, b\})$ is good if it has a corresponding good tree-representation with vertices $a$ and $b$, and bad if none exists.

Lemma 3.5. Let $G$ be a graph and let $a, b \in V(G)$. Let $H$ be the graph obtained from $G$ by adding two new vertices, say $c$ and $d$, and edges $a c, c d$ and $d b$, followed by removing the edge $a b$ if $a b \in E(G)$. If $(G,\{a, b\})$ is bad, then $\operatorname{mmw}(H) \geq 3$.

Proof. We prove by contradiction. Suppose $\operatorname{mmw}(H) \leq 2$, that is, $H$ has a tree-representation $\mathcal{T}=\left(T,\left\{T_{v}\right\}_{v \in V(H)}\right)$ of width at most 2. We shall use $\mathcal{T}$ to find a good tree-representation of $G$ with $a$ and $b$, yielding a contradiction.

From $\mathcal{T}$ we may obtain three tree-representations of $G$ with width at most 2 by replacing the subtree for $a$ and $b$ respectively with (1) $T_{a} \cup T_{c} \cup T_{d}$ and $T_{b}$, (2) $T_{a} \cup T_{c}$ and $T_{d} \cup T_{b}$, and (3) $T_{a}$ and $T_{c} \cup T_{d} \cup T_{b}$. Since $(G,\{a, b\})$ is bad, for all three choices the subtrees for $a$ and $b$ intersect at precisely one vertex in the new tree-representations. Therefore, $E\left(T_{a}\right) \cap E\left(T_{b}\right)=\emptyset$ and $T$ has two distinct vertices $v_{1}$ and $v_{2}$ such that $T_{a} \cap T_{c}=\left\{v_{1}\right\}$ and $T_{d} \cap T_{b}=\left\{v_{2}\right\}$.

Let $e=v_{1} u$ be the first edge in the unique path $P$ in $T$ from $v_{1}$ to $v_{2}$. Because of the path $a c d b$ in $H$, the first few consecutive edges of $P$ (possibly zero) are in $T_{c}$ and the others are in $T_{d}$. We assume that $T_{c}$ contains $e$. The following manipulation can be done likewise when $T_{d}$ contains $e$.

Let $x \neq c$ be a vertex of $G$ such that $T_{x}$ contains $e$. If there is no such $x$ we ignore $x$ in the following. Let $T^{\prime}$ be the tree obtained from $T$ by subdividing $e$, and adding a path $v_{3} v_{4} v_{5}$ of length 2 at the new vertex $v_{3}$ obtained from the subdivision; see Fig. 2. Let $\left\{T_{v}^{\prime}\right\}_{v \in V(H)}$ be a collection of subtrees of $T^{\prime}$ such that

- $T_{c}^{\prime}$ and $T_{d}^{\prime}$ have only one edge $v_{4} v_{5}$,

- $T_{a}^{\prime}$ is obtained from $T_{a}$ by adding the edges $v_{1} v_{3}$ and $v_{3} v_{4}$,

- $T_{b}^{\prime}$ is obtained from $T_{b}$ by adding $v_{3} v_{4}$ and the edges on the path from $v_{3}$ to $v_{2}$, and

- $T_{v}^{\prime}=T_{v}$ for all other $v \in V(H)$. 

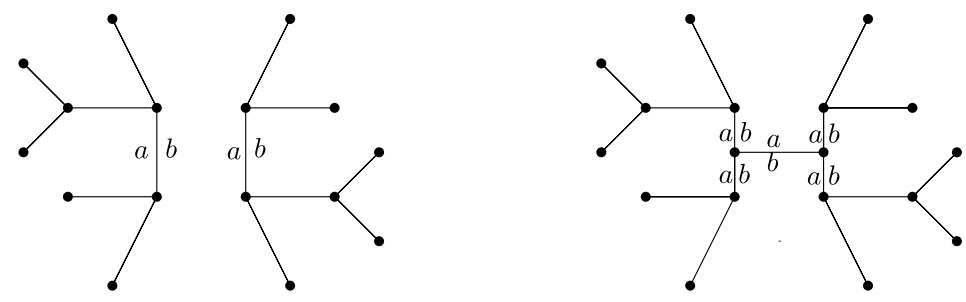

Fig. 3. New tree-representation from two tree-representations.

Note that for each pair of vertices $u, v$ in $V(H) \backslash\{c, d\}$, if $T_{u} \cap T_{v} \neq \emptyset$ then $T_{u}^{\prime} \cap T_{v}^{\prime} \neq \emptyset$. Since $c$ and $d$ are adjacent to only $a$ and $b$ in $H$, the pair $\mathcal{T}^{\prime}=\left(T^{\prime},\left\{T_{v}^{\prime}\right\}_{v \in V(H)}\right)$ is a tree-representation of $H$ of width 2 . Hence, by removing $v_{5}$, we obtain a good tree-representation of $G$ with $a, b$ having width 2 , a contradiction.

Lemma 3.6. Let $G$ be a graph and let $c$ be a vertex of $G$ with precisely two neighbors $a$ and $b$. If $a b \in E(G)$ and $m m w(G) \geq 3$, then $\operatorname{mmw}(G \backslash a b) \geq 3$.

Proof. We prove by contradiction. Suppose $H=G \backslash a b$ has a tree-representation $\mathcal{T}=\left(T,\left\{T_{v}\right\}_{v \in V(H)}\right)$ of width at most 2 . Since $\operatorname{mmw}(G) \geq 3$ the subtrees $T_{a}$ and $T_{b}$ are vertex-disjoint. Let $v_{1} \in V\left(T_{a}\right)$ and $v_{2} \in V\left(T_{b}\right)$ be the vertices of $T$ such that the unique path $P$ in $T$ from $v_{1}$ to $v_{2}$ have no edge in neither $T_{a}$ nor $T_{b}$. As $c$ is a common neighbor of $a$ and $b$, every edge of $P$ is in $T_{c}$. Now we do the same as in the proof of Lemma 3.5 and Fig. 2, except that here we set $d=c$. The resulting tree-representation of $G$ has width at most 2, a contradiction.

A 2-cut in $G$ is an inclusion-wise minimal subset $S \subset V(G)$ such that $|S|=2$ and $G \backslash S$ is disconnected. Given a graph $G$ and its 2-cut $\{a, b\}$ with a component $S$ of $G \backslash\{a, b\}$, we denote by $\tilde{S}$ the induced subgraph $G[V(S) \cup\{a, b\}]$. As $\{a, b\}$ is the unique 2 -cut having $S$ as a component, we may say simply $\tilde{S}$ is good or bad, if respectively the pair $(\tilde{S},\{a, b\})$ is good or bad.

Lemma 3.7. Let $G$ be a graph in $\mathcal{O}_{2}$. If a 2-cut $\{a, b\}$ separates $G$ into two components $A$ and $B$, then $a b \notin E(G)$ and one of $\tilde{A}$ or $\tilde{B}$ is isomorphic to either $P_{3}$ or $P_{4}$.

Proof. We start with showing that one of $\tilde{A}$ and $\tilde{B}$ is bad. Suppose for contradiction that both are good. From their good representations, say $\left(T^{A},\left\{T_{x}\right\}_{x \in V(A)}\right)$ and $\left(T^{B},\left\{T_{y}\right\}_{y \in V(B)}\right)$, we can construct a tree-representation of $G$ of width 2 as follows. We choose an edge from each of $T^{A}$ and $T^{B}$ shared by $T_{a}$ and $T_{b}$, and then subdivide those two edges and connect the new vertices by an edge; see Fig. 3. The new subtrees $T_{a}^{\prime}$ and $T_{b}^{\prime}$ will be clear from Fig. 3. It is easy to see that the resulting tree-representation has width 2 .

Now we assume $\tilde{B}$ is bad. Suppose $|A| \geq 2$. Since $G$ is 2-connected, there exists a path from $a$ to $b$ in $\tilde{A}$ whose length is at least 3. Thus, $\tilde{A}$ contains $P_{4}$ as a minor. Let $H$ be a graph obtained from $\tilde{B}$ and $P_{4}$ by identifying $a$ and $b$ in $\tilde{B}$ with two ends of $P_{4}$ respectively. By Lemma 3.5, $H$ has mm-width at least 3. If $P_{4}$ is a proper minor of $\tilde{A}$, then a graph $H$ is a proper minor of $G$. It contradicts that $G$ is in $\mathcal{O}_{2}$. Therefore, $\tilde{A}$ is isomorphic to $P_{4}$.

Suppose $A=\{c\}$. Since $G$ is 2-connected, $c$ is adjacent to both $a$ and $b$ and by Lemma 3.6, $\tilde{A}$ is the path $a c b$, which is isomorphic to $P_{3}$.

To consider the 2-cuts with more than two components, we use the following lemma. The proof of Lemma 3.5 can be modified to prove the following.

Lemma 3.8. Let $G$ be a graph and let $a, b \in V(G)$. Let $H$ be the graph obtained from $G$ by adding two new vertices, say $c$ and $d$, and edges $a c, b c$, ad and $b d$, followed by removing the edge $a b$ if $a b \in E(G)$. If $(G,\{a, b\})$ is bad, then $\operatorname{mmw}(H) \geq 3$.

Let $G \in \mathcal{O}_{2}$. Suppose that a 2 -cut $\{a, b\}$ separates $G$ into at least three components, namely $D_{1}, D_{2}, \ldots, D_{k}$. Since we can combine arbitrary number of good tree-representations as in Fig. 3 while preserving goodness, one of the $\tilde{D}_{i}$ 's, say $\tilde{D}_{1}$, is bad. If $k>3$, we construct $G^{\prime}$ by contracting each of $D_{2}, \ldots, D_{k}$ into a single vertex, and leaving just two of those. Then by Lemma 3.8, $\mathrm{mmw}\left(G^{\prime}\right) \geq 3$. Therefore, $G$ cannot contain $G^{\prime}$ as a proper minor. Also by Lemma $3.7, \tilde{D}_{i}$ is isomorphic to either $P_{3}$ or $P_{4}$ for $2 \leq i \leq k$. As a result, one of the following holds:

1. $k=2$ and $\tilde{D}_{2}=P_{3}$.

2. $k=2$ and $\tilde{D}_{2}=P_{4}$.

3. $k=3$ and $\tilde{D}_{2}=\tilde{D}_{3}=P_{3}$.

We summarize the above discussion as follows.

Corollary 3.9. Let $G$ be a graph in $\mathcal{O}_{2}$. If $\{a, b\}$ is a 2-cut of $G$, then $G \backslash\{a, b\}$ has a unique component $B_{a b}$ such that $\tilde{B}_{a b}=G\left[V\left(B_{a b}\right) \cup\{a, b\}\right]$ is bad. Moreover, $a b \notin E(G)$ and $G \backslash B_{a b}$ is one of the following: 


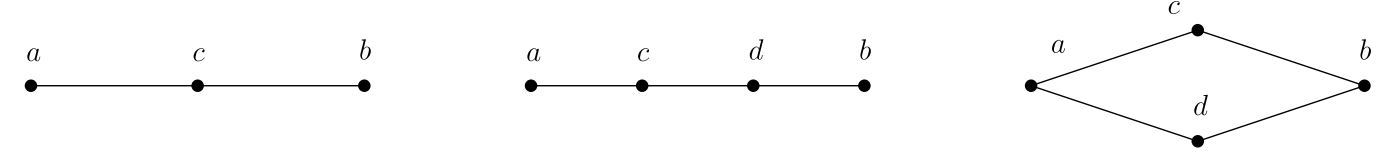

Fig. 4. Three ways of replacing an edge $a b$.

- a path of length 2 between $a$ and $b$,

- a path of length 3 between $a$ and $b$, or

- $a K_{2,2}$ where $a$ and $b$ are non-adjacent.

Following Corollary 3.9, for each 2-cut $\{a, b\}$ of $G \in \mathcal{O}_{2}$, we call $G \backslash B_{a b}$ the good-side of $\{a, b\}$. Hence the good-side of $\{a, b\}$ contains at most two vertices other than $a$ and $b$.

We shall show below that every graph in $\mathcal{O}_{2}$ can be constructed from a small 3-connected graph by replacing some of its edges by some of the three graphs in Fig. 4. To state this precisely, we call the replacement of an edge $a b$ with $P_{3}=a c b$, $P_{4}=a c d b$ and $K_{2,2}=a c b \cup a d b$, respectively, as 1-subdivision, 2-subdivision and 11-subdivision where $c, d$ are adjacent to no other vertices; see Fig. 4. We call these three operations as good-subdivisions.

Lemma 3.10. Every graph in $\mathrm{O}_{2}$ is obtained from a 3-connected graph on 4, 5, or 6 vertices by good-subdividing some of its edges.

Proof. Let us consider the inclusion-wise maximal good-sides of 2-cuts. We would like to replace each of them with an edge between the vertices in its 2-cut. To make this operation valid, we begin with showing that if two good-sides intersect, then both of them are contained in a good-side that is $P_{4}$, or the intersection is a single vertex contained in both of their 2-cuts. Note that if $\tilde{A}$ is bad then $A$ has at least 5 vertices, as $K_{6}$ has a good tree-representation for every pair of its vertices.

Let $G$ be a graph in $\mathcal{O}_{2}$. Let $\{a, b\}$ be a 2-cut in $G$ and let $B_{a b}$ be the unique component of $G \backslash\{a, b\}$, by Corollary 3.9, such that $\tilde{B}_{a b}=G\left[V\left(B_{a b}\right) \cup\{a, b\}\right]$ is bad. Let $\{c, d\}$ be another 2-cut in $G$ and suppose that $c$ is in $G \backslash \tilde{B}_{a b}$. By Corollary 3.9, $c$ has only two neighbors in $G$ and is in a path connecting $a$ and $b$ of length 2 or 3 . We claim that if $d$ is in $B_{a b}$, then either

- $\{a, d\}$ is a 2-cut of $G$ with its good-side being the path $a c b d$, or

- $\{b, d\}$ is a 2-cut of $G$ with its good-side being the path bcad.

That is, if the good-sides of $\{a, b\}$ and $\{c, d\}$ intersect nontrivially, then both are contained in a larger good-side of another 2-cut.

Now we prove the claim. Suppose that $d$ is in $B_{a b}$. Note that $c$ is a cut-vertex of $G \backslash d$ and $c$ has only two neighbors in $G \backslash d$ (same as in $G$ ), one is connected to $a$ and the other is connected to $b$. Hence the graph $G \backslash\{c, d\}$ does not have a path between $a$ and $b$, so that its subgraph $\tilde{B}_{a b} \backslash d$ does not have one either. Thus $d$ is a cut-vertex of $\tilde{B}_{a b}$ separating $a$ and $b$. Moreover, $\tilde{B}_{a b} \backslash d$ has precisely two components, whose vertex sets we call $D_{a}$ and $D_{b}$ with $a \in D_{a}$ and $b \in D_{b}$; otherwise $G$ is not 2-connected.

By Corollary 3.9, $a b \notin E(G)$ and $c d \notin E(G)$. Suppose that $D_{a}=\{a\}$. Since $\tilde{B}_{a b}$ contains at least seven vertices, the set $\{d, b\}$ must be a 2-cut of $G$. The good-side of $\{d, b\}$, by counting the vertices, must be the component containing $c$ and by Corollary 3.9 we deduce that dacb is an induced path in $G$ and $a, c$ have no other neighbors, which is what we claimed. Similarly we can settle the case when $D_{b}=\{b\}$.

Thus we may assume that $\left|D_{a}\right| \geq 2$ and $\left|D_{b}\right| \geq 2$. Since $\{c, d\}$ is a 2-cut of $G$, by Corollary 3.9, we have either $\left|D_{a}\right|=2$ or $\left|D_{b}\right|=2$. We assume $\left|D_{b}\right|=2$. As $D_{a} \cup D_{b} \cup\{d\}$ constitutes $\tilde{B}_{a b}$, we have $\left|D_{a}\right|+\left|D_{b}\right|+1 \geq 7$, so that $\left|D_{a}\right| \geq 4$. Now we consider the 2-cut $\{a, d\}$. The component containing $c$ and $b$ has at least three vertices, so this component must be $B_{a d}$. But $\left|D_{a}\right| \geq 4$ implies $G \backslash \widetilde{B}_{a d}$ having at least three vertices, a contradiction to Corollary 3.9. This proves the claim.

For a 2-cut $\{a, b\}$ of $G$, we denote by $C_{a b}=G \backslash B_{a b}$ the good-side of $\{a, b\}$. What we showed above is that, if $C_{a b} \cap C_{c d} \neq \emptyset$ for some $\{c, d\}$ then either (1) $C_{a b} \subseteq C_{c d}$, (2) $C_{c d} \subseteq C_{a b}$, or (3) the intersection consists of a single vertex which is one of $a, b, c, d$.

Now we replace the good-sides at once by edges between their cuts. Formally, let $\mathcal{C}=\left\{C_{a b}:\right.$ is a 2-cut of $\}$ a,b $\}$ G. Let $\mathcal{C}^{*} \subseteq \mathcal{C}$ be the subset of inclusion-wise maximal elements. For each $C_{a b} \in \mathcal{C}^{*}$, we remove $C_{a b} \backslash\{a, b\}$ from $G$ and add an edge $a b$ to $G$. This operation is well-defined because of the above discussion. Let $H$ be the resulting proper minor of $G$. If $H$ has a 2-cut, then we construct $G$ back from $H$ and the 2-cut still remains in $G$, which is impossible since for each 2-cut $S$, we remove all but one component of $G \backslash S$ while producing $H$. That is, $H$ is either 3-connected or isomorphic to $K_{2}$ or $K_{3}$.

If $H$ has at least 7 vertices, then $H$ has a minor in $\mathcal{O}_{3}$ so that $G \notin \mathcal{O}_{2}$. Thus $H$ has at most 6 vertices. If $H$ is $K_{2}$, then $H$ is constructed by connecting several good-sides in parallel, which has mm-width at most 2 as shown in the proof of Lemma 3.7, especially Fig. 3. If $H$ is a triangle $a b c$, then $G$ is obtained from $a b c$ by good-subdividing all three edges $a b, b c$ and $c a$. To find a tree-representation of $G$ with width 2 in this case, we start from a $K_{1,3}$ where its three edges are labeled respectively by $a b, b c$ and $c a$. Then we can add the good-sides for the edges $a b, b c$ and $c a$ without increasing the width. Hence $H$ has 4 , 5, or 6 vertices and is 3 -connected.

The obstructions obtained from a 3-connected graph on 4, 5, and 6 vertices respectively are listed in Figs. 5, 6 and 7. The respective proofs are given in Lemmas 3.14, 3.15 and 3.16. Note that Lemmas 3.5 and 3.8 imply the following. 


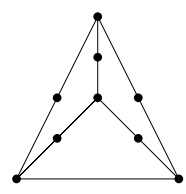

(a) $\mathcal{O}_{4}^{1}$.

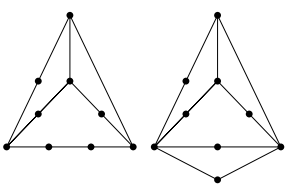

(b) $\mathcal{O}_{4}^{2}$.
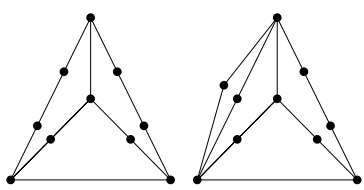

(c) $\mathcal{O}_{4}^{3}$.

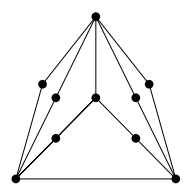

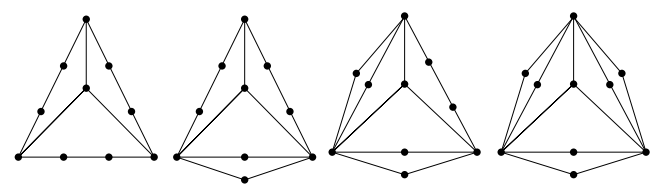

(d) $\mathcal{O}_{4}^{4}$.

Fig. 5. The graphs in $\mathcal{O}_{4}=\mathcal{O}_{4}^{1} \cup \mathcal{O}_{4}^{2} \cup \mathcal{O}_{4}^{3} \cup \mathcal{O}_{4}^{4}$.

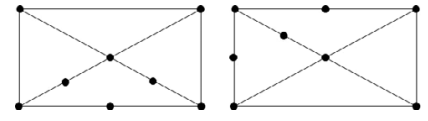

(a) $\mathcal{O}_{5}^{1}$.

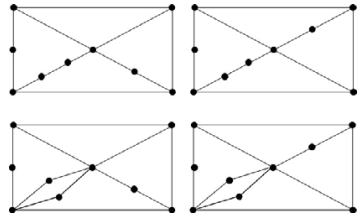

(d) $\mathcal{O}_{5}^{4}$.

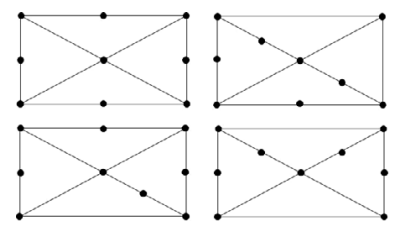

(b) $\mathcal{O}_{5}^{2}$.
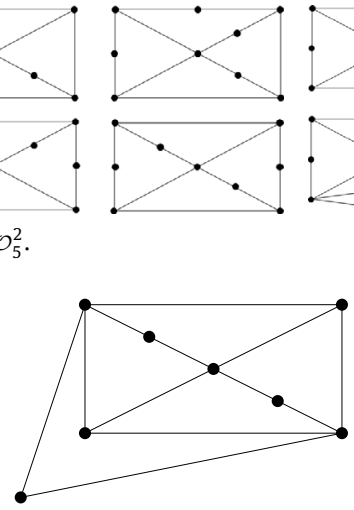

(e) $\mathcal{O}_{5}^{5}$.

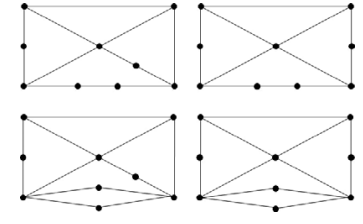

(c) $\mathcal{O}_{5}^{3}$.

Fig. 6. The graphs in $\mathcal{O}_{5}=\mathcal{O}_{5}^{1} \cup \mathcal{O}_{5}^{2} \cup \mathcal{O}_{5}^{3} \cup \mathcal{O}_{5}^{4} \cup \mathcal{O}_{5}^{5}$.

Lemma 3.11. Let $G$ be a graph with an induced path acdb such that $c$ and $d$ are non-adjacent to other vertices. Let $H$ be the graph obtained from $G-\{c, d\}$ by adding two new vertices $c^{\prime}, d^{\prime}$ and paths $a c^{\prime} b$ and $a d^{\prime} b$. Then $G \in \mathcal{O}_{2}$ if and only if $H \in \mathcal{O}_{2}$.

Hence in the following discussion we do not consider 11-subdivisions. The obstructions obtained by replacing 2subdivisions with 11-subdivisions shall be added to the list without mentioning.

We shall use the following lemma often when we show a graph has mm-width at most 2.

Lemma 3.12. Let $G$ be a graph. If $\left\{V_{1}, V_{2}, V_{3}\right\}$ is a partition of $V(G)$ and $G$ has six vertices $a_{i}, b_{i}$ for $i=1,2,3$ such that for each $i$,

(1) $a_{i}, b_{i} \in V_{i}$,

(2) $\left\{a_{i}, b_{i}\right\}$ separates $V_{i}$ from $V(G) \backslash V_{i}$, and

(3) $\left(G\left[V_{i}\right],\left\{a_{i}, b_{i}\right\}\right)$ has a good tree-representation,

then $\operatorname{mmw}(G) \leq 2$.

Proof. For each $i$, we consider a good tree-representation of $G\left[V_{i}\right]$ such that the subtrees for $a_{i}$ and $b_{i}$ share an edge whose one end has degree 1 . We combine the three tree-representations by identifying those degree 1 vertices to obtain a treerepresentation of $G$ with width at most 2 .

The way we use Lemma 3.10 to show a graph has mm-width $\leq 2$ is that, we try to cover the graph with either three good-sides or two good-sides and a set of at most two vertices. If we do so, the sets become $V_{1}, V_{2}$ and $V_{3}$ in the statement and Lemma 3.10 applies.

For convenience, we state here at once that the graphs in the following Lemmas 3.14-3.16 all have mm-width at least 3. Lemma 2.6 is a corollary of the following lemma. 


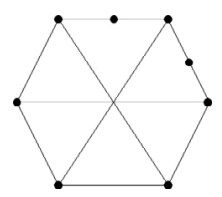

(a) $\mathcal{O}_{6}^{1}$.

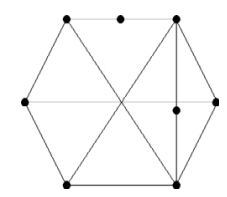

(b) $\mathcal{O}_{6}^{2}$.

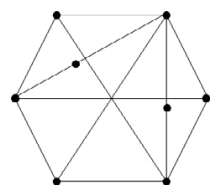

(c) $\mathcal{O}_{6}^{3}$.

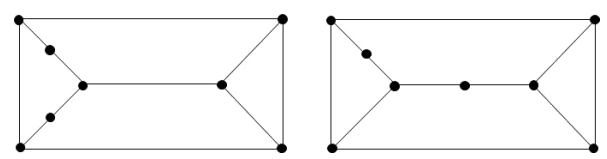

(d) $\mathcal{O}_{6}^{4}$.
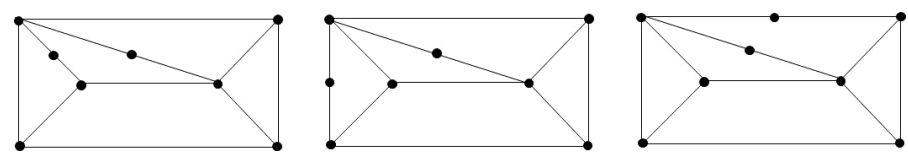

(e) $\mathcal{O}_{6}^{5}$.
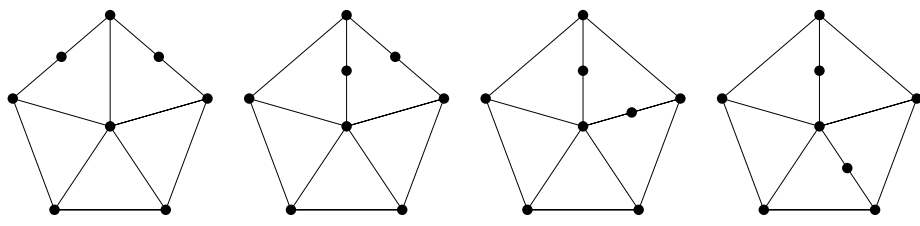

(f) $\mathcal{O}_{6}^{6}$.

Fig. 7. The graphs in $\mathcal{O}_{6}=\mathcal{O}_{6}^{1} \cup \mathcal{O}_{6}^{2} \cup \mathcal{O}_{6}^{3} \cup \mathcal{O}_{6}^{4} \cup \mathcal{O}_{6}^{5} \cup \mathcal{O}_{6}^{6}$.

Lemma 3.13. Let $\mathcal{O}_{4}, \mathcal{O}_{5}$ and $\mathcal{O}_{6}$, respectively, be the set of graphs in Figs. 5-7. Every graph in $\mathcal{O}_{4} \cup \mathcal{O}_{5} \cup \mathcal{O}_{6}$ has maximum matching width at least 3.

Proof. By Theorem 2.5, it is enough to give a tangle of order 3. We shall explain how to find a tangle of order 3 for each of those graphs.

Let $G$ be a graph and let $X$ be a subset of $V(G)$ such that $m m(X) \leq 2$ and $|X| \geq 3$. In other words, the bipartite graph on $V(G)$ with all the edges in $E(G)$ having one end in $X$ and the other not in $X$ has maximum matching size 2 . Thus we can find a set $\{a, b\}$ that is a 2 -cut of $G$ and $G \backslash\{a, b\}$ has a component, say $S$, such that $\tilde{S}$ contains either $X$ or $V(G) \backslash X$.

Therefore, for each graph $G \in \mathcal{O}_{4} \cup \mathcal{O}_{5} \cup \mathcal{O}_{6}$, we set $S_{G}$ to be the collection of all vertex subsets of the following three types:

- a set of size at most 2

- a good-side of a 2-cut

- if $G$ has a 11 -subdivision made of the paths $a u b$ and $a v b$, then $S_{G}$ contains both $\{a, u, b\}$ and $\{a, v, b\}$.

Now we consider the tangle axioms (T1), (T2) and (T3) in Section 2.2 to verify that $S_{G}$ is a tangle. (T1) follows immediately from the above discussion, and (T3) is also easy to check for all graphs in $G \in \mathcal{O}_{4} \cup \mathcal{O}_{5} \cup \mathcal{O}_{6}$. For (T2), we can check that no three good-sides cover the whole graph and it remains to see that there are no two good-sides that cover all but at most two vertices. We leave the details to the reader.

Now we consider the case when the 3-connected graph in Lemma 3.10 has four vertices. The only 3-connected graph on four vertices is $K_{4}$.

Lemma 3.14. Let $\mathcal{O}_{4}^{1}, \mathcal{O}_{4}^{2}, \mathcal{O}_{4}^{3}, \mathcal{O}_{4}^{4}$ be the sets of graphs in Fig. 5. If a graph $\mathrm{G}$ is obtained from $\mathrm{K}_{4}$ by good-subdividing some of its edges, then $G \in \mathcal{O}_{2}$ if and only if $G \in \mathcal{O}_{4}=\mathcal{O}_{4}^{1} \cup \mathcal{O}_{4}^{2} \cup \mathcal{O}_{4}^{3} \cup \mathcal{O}_{4}^{4}$.

Proof. By Lemma 3.13 the graphs in $\mathcal{O}_{4}$ have mm-width at least 3. It can be easily checked that all their proper minors have mm-width at most 2 using Lemma 3.12.

Now we consider the graphs obtained from $K_{4}$ by good-subdivisions. We divide the cases via the number of goodsubdivisions. Recall that by Lemma 3.11, we only consider 2-subdivision and not 11-subdivision.

If $G$ has no 2 -subdivision and has at most four 1-subdivisions, then $\operatorname{mmw}(G) \leq 2$ by Lemma 3.12. The unique graph with no 2 -subdivision and five 1 -subdivisions is in $\mathcal{O}_{4}^{1}$.

If $G$ has one 2-subdivision and at most three 1-subdivisions, then $\operatorname{mmw}(G) \leq 2$ by Lemma 3.12 unless $G$ is the first graph in $\mathcal{O}_{4}^{2}$. If $G$ has one 2-subdivision and four 1-subdivisions, then $G$ contains the graph in $\mathcal{O}_{4}^{1}$ as a minor.

If $G$ has two 2-subdivisions and at most two 1-subdivisions, then either $G$ has mm-width 2, $G$ contains a graph in $\mathcal{O}_{4}^{2}$ as a minor, or $G$ is the first graph in $\mathcal{O}_{4}^{3}$. The rest of $\mathcal{O}_{4}^{3}$ is obtained by replacing 2-subdivisions with 11-subdivisions; see Lemma 3.11. If $G$ has more than two 1-subdivisions, then $G$ contains the graph in $\mathcal{O}_{4}^{1}$ as a minor. 
If $G$ has three 2-subdivisions and no 1 -subdivision, then $\operatorname{mmw}(G) \leq 2$ by Lemma 3.12 unless $G$ is the first graph in $\mathcal{O}_{4}^{4}$. If $G$ has three 2-subdivisions and at least one 1-subdivision, then $G$ contains a graph in $\mathcal{O}_{4}^{2} \cup \mathcal{O}_{4}^{3}$ as a minor.

Lemmas 3.15 and 3.16, respectively, characterize the graphs in $\mathrm{O}_{2}$ that are obtained from a 3-connected graph on five and six vertices.

Lemma 3.15. Let $\mathcal{O}_{5}^{1}, \mathcal{O}_{5}^{2}, \mathcal{O}_{5}^{3}, \mathcal{O}_{5}^{4}, \mathcal{O}_{5}^{5}$ be the sets of graphs in Fig. 6. If a graph $\mathrm{G}$ is obtained from a 3-connected graph on 5 vertices by good-subdividing some edges, then $G \in \mathcal{O}_{2}$ if and only if $G \in \mathcal{O}_{5}=\mathcal{O}_{5}^{1} \cup \mathcal{O}_{5}^{2} \cup \mathcal{O}_{5}^{3} \cup \mathcal{O}_{5}^{4} \cup \mathcal{O}_{5}^{5}$.

Proof. By Lemma 3.13 the graphs in $\mathcal{O}_{5}$ have mm-width at least 3 . Their proper minors have mm-width 2 by Lemma 3.12 and hence they are in $\mathrm{O}_{2}$.

Now we consider the graphs that are also obtained from a 3-connected graph on 5 vertices by good-subdivisions. There are three 3-connected graphs on 5 vertices, namely the wheel $W_{5}, W_{5}$ plus an edge (say $W_{5}^{\prime}$ ), and $K_{5}$.

Let us begin with $W_{5}$. Let $G$ be a graph obtained from $W_{5}$ by good-subdividing some edges.

Suppose that $G$ has no 2 -subdivision and has three 1 -subdivisions. If the to-be-subdivided edges of $W_{5}$ contain two independent edges, then Lemma 3.12 implies $\operatorname{mmw}(G) \leq 2$. Thus $G \in \mathcal{O}_{2}$ if and only if $G \in \mathcal{O}_{5}^{1}$. If $G$ has no 2-subdivision and has four 1-subdivisions, then it has mm-width 3; a tangle of order 3 can be found in each case as in Lemma 3.13. So in this case $G \in \mathcal{O}_{2}$ if and only if it does not have a graph in $\mathcal{O}_{5}^{1}$ as a minor. These are the graphs in $\mathcal{O}_{5}^{2}$.

If the number of 1-subdivisions and 2-subdivisions in $G$ is at least 4, then $G$ contains a graph in $\mathcal{O}_{5}^{1} \cup \mathcal{O}_{5}^{2}$ as a minor. Suppose $G$ has one 2-subdivision and two 1-subdivisions. If the good-side of the 2-subdivision does not intersect with one of the other two good-sides, then Lemma 3.12 implies $\operatorname{mmw}(G) \leq 2$. Thus both good-sides of the 1 -subdivisions intersect with the good-side of the 2-subdivision. If the 2-subdivision happens at an edge incident with the vertex of degree 4 in $W_{5}$, then $G \in \mathcal{O}_{2}$ if and only if $G$ is one of the top two graphs in $\mathcal{O}_{5}^{4}$; other cases contain a graph in $\mathcal{O}_{5}^{1}$ as a minor. The bottom two graphs in $\mathcal{O}_{5}^{4}$ are obtained by replacing the 2-subdivision with a 11-subdivision. If the 2-subdivision is not incident with the degree- 4 vertex of $W_{5}$, then we get the graphs in $\mathcal{O}_{5}^{3}$.

If $G$ has at least two 2 -subdivisions, then either $\operatorname{mmw}(G) \leq 2$ or it contains a graph in $\mathcal{O}_{5}^{1} \cup \mathcal{O}_{5}^{2} \cup \mathcal{O}_{5}^{3} \cup \mathcal{O}_{5}^{4}$ as a minor. It completes the graphs obtained from $W_{5}$.

Now we consider the graphs $G \in \mathcal{O}_{2}$ obtained from $W_{5}^{\prime}$ by good-subdivisions. The graph $W_{5}^{\prime}$ has three edges whose removal results in $W_{5}$. Suppose one of these three edges, say $e$, is not good-subdivided in $G$. If $G$ has at least four goodsubdivisions, then $G-e$ contains a graph in $\mathcal{O}_{5}^{1} \cup \mathcal{O}_{5}^{2} \cup \mathcal{O}_{5}^{3} \cup \mathcal{O}_{5}^{4}$ as a minor. If $G$ has at most three good-subdivisions and $G-e$ does not contain a graph in $\mathcal{O}_{5}^{1}$ as a minor, then Lemma 3.12 implies $\operatorname{mmw}(G) \leq 2$. Hence all three edges of $W_{5}^{\prime}$ in the triangle of degree-4 vertices must be good-subdivided in $G$. Since the graph in $\mathcal{O}_{5}^{5}$ is in $\mathcal{O}_{2}$, it is the unique graph obtained from $W_{5}^{\prime}$ in $\mathcal{O}_{2}$.

The last 3-connected graph on five vertices is $K_{5}$. Let $G$ be a graph obtained from $K_{5}$ by good-subdivisions. Using an argument similar to above we can show that every edge of $K_{5}$ must be subdivided. Hence $G \notin \mathcal{O}_{2}$ and $\mathcal{O}_{5}$ is the precise set of obstructions obtained from a 3-connected graph on five vertices.

Lemma 3.16. Let $\mathcal{O}_{6}^{1}, \mathcal{O}_{6}^{2}, \mathcal{O}_{6}^{3}, \mathcal{O}_{6}^{4}, \mathcal{O}_{6}^{5}, \mathcal{O}_{6}^{6}$ be the sets of graphs in Fig. 7. If a graph $G$ is obtained from a 3-connected graph on 6 vertices by good-subdivisions, then $G \in \mathcal{O}_{2}$ if and only if $G \in \mathcal{O}_{6}=\mathcal{O}_{6}^{1} \cup \mathcal{O}_{6}^{2} \cup \mathcal{O}_{6}^{3} \cup \mathcal{O}_{6}^{4} \cup \mathcal{O}_{6}^{5} \cup \mathcal{O}_{6}^{6}$.

Proof. Let $H$ be a 3-connected graph on six vertices and let $G$ be a graph obtained from $H$ by good-subdividing some edges. If two adjacent edges of $H$ are good-subdivided in $G$, then we can find a tangle of order 3 in $G$ and hence mmw( $G) \geq 3$; all graphs in $\mathcal{O}_{6}$ are of this type. If there is no such pair in $H$, then the good-subdivisions happened at a matching of $H$ and Lemma 3.12 implies $\operatorname{mmw}(G) \leq 2$. We leave it to the reader to check that the proper minors of the graphs in $\mathcal{O}_{6}$ have mm-width at most 2 .

If $H$ is minimally 3-connected, then all the graphs obtainable from $H$ by good-subdividing two adjacent edges are in $\mathcal{O}_{6}$; $\mathcal{O}_{6}^{1}$ for $K_{3,3}, \mathcal{O}_{6}^{4}$ for the prism and $\mathcal{O}_{6}^{6}$ for the wheel $W_{6}$.

Suppose that $H$ is not minimally 3-connected and $G \in \mathcal{O}_{2}$. Let $e$ be an edge of $H$ such that $H-e$ is still 3-connected. If $e$ is not subdivided in $G$, then by the above discussion $G-e$ has two adjacent good-sides and $\operatorname{mmw}(G-e) \geq 3$, a contradiction. Thus $e$ must be good-subdivided in $G$ and $H$ has at most two edges whose removal does not affect its 3-connectivity. Note that if $H$ has two such edges, then they should be also adjacent.

If $H$ is $K_{3,3}$ plus an edge, then the additional edge must be subdivided and we need another adjacent edge to subdivide. But independently of this choice the resulting graph is isomorphic to the graph in $\mathcal{O}_{6}^{2}$. There is a unique way of adding two adjacent edges to $K_{3,3}$ and the graph in $\mathcal{O}_{6}^{3}$ is the result of subdividing both.

If $H$ is the prism plus an edge, then we have three non-isomorphic choices of another adjacent edge to subdivide. They are in $\mathcal{O}_{6}^{5}$. There is again a unique way of adding two adjacent edges to the prism but it contains a graph in $\mathcal{O}_{6}^{6}$ as a minor.

There is a unique (up to isomorphism) way to add an edge to $W_{6}$ but it already has three edges that are removable while maintaining 3-connectivity. Thus the list is complete.

By Lemma 3.4, a graph is in the obstruction set and 3-connected if and only if it is in $\mathcal{O}_{3}$. If $G$ is in the obstruction set but not 3-connected, then it should be obtained from a 3-connected graph on 4, 5, or 6 vertices by Lemma 3.10. Lemmas 3.14-3.16 show that $G \in \mathcal{O}_{4} \cup \mathcal{O}_{5} \cup \mathcal{O}_{6}$. Therefore, the following theorem holds: 
Theorem 3.17. Let $\mathcal{O}=\mathcal{O}_{3} \cup \mathcal{O}_{4} \cup \mathcal{O}_{5} \cup \mathcal{O}_{6}$ be the set of 45 graphs in Figs. 1, 5-7. A graph $G$ has maximum matching width at most 2 if and only if $G$ has no minor isomorphic to a graph in $\mathcal{O}$.

\section{4. $k \times k$-grid}

The $k \times k$-grid, denoted by $G_{k}$, is the graph with a vertex set $V\left(G_{k}\right)=\{(i, j): 1 \leq i, j \leq k\}$ and an edge set $E\left(G_{k}\right)=\left\{(i, j)\left(i^{\prime}, j^{\prime}\right):\left|i-i^{\prime}\right|+\left|j-j^{\prime}\right|=1\right\}$. In this section, we show $m m w\left(G_{k}\right)=k$ for $k \geq 2$.

Vatshelle [20] showed the following inequality. Recall that $\operatorname{rw}(G)$ and brw $(G)$ respectively denote the rank-width and the branch-width of $G$.

Theorem 4.1 ([20]). If $G$ is a graph, then

$\operatorname{rw}(G) \leq \operatorname{mmw}(G) \leq \max (\operatorname{brw}(G), 1)$.

It is known that $\operatorname{brw}\left(G_{k}\right)=k$ [12] and $\operatorname{rw}\left(G_{k}\right)=k-1$ [7]. Hence $\operatorname{mmw}\left(G_{k}\right)$ is either $k-1$ or $k$. We shall show $\operatorname{mmw}\left(G_{k}\right)>k-1$ by finding a tangle of order $k$; see Section 2.2. We assume $k \geq 2$ throughout this section.

Let $C_{i}=\{(i, j): 1 \leq j \leq k\}$ and $R_{j}=\{(i, j): 1 \leq i \leq k\}$ be the set of vertices on the $i$-th column and the $j$-th row respectively. Recall that for a vertex set $X \subseteq V(G), \operatorname{mm}_{G}(X)$ denotes the size of a maximum matching in $G[X, V(G) \backslash X]$. We omit $G_{k}$ in $\mathrm{mm}_{G_{k}}$ and write $\mathrm{mm}(X)=\mathrm{mm}_{G_{k}}(X)$ in this section. Let $X^{c}=V\left(G_{k}\right) \backslash X$ for $X \subseteq V\left(G_{k}\right)$.

Lemma 4.2. If $X \subseteq V\left(G_{k}\right)$ and $\mathrm{mm}(X)<k$, then $R_{i} \subseteq X$ for some $i$ if and only if $C_{j} \subseteq X$ for some $j$.

Proof. Suppose that $R_{i} \subseteq X$ for some $i$. Then each $C_{j}$ intersects with $X$. If $C_{j} \nsubseteq \subseteq X$ for every $j$, each $G\left[C_{j}\right]$ contains an edge with one end in $X$ and the other end in $X^{c}$. Since these edges form a matching of size $k$, we have $\operatorname{mm}(X) \geq k$ which is a contradiction. Thus $C_{j} \subseteq X$ for some $j$. The converse follows from the symmetry.

For $X \subseteq V\left(G_{k}\right)$, we say that $X$ is small if $\mathrm{mm}(X)<k$ and $R_{i} \nsubseteq X$ for all $i=1,2, \ldots, k$. Note that, by Lemma 4.2, $C_{j} \nsubseteq X$ for all $j=1,2, \ldots, k$ if $X$ is small.

Lemma 4.3. Let $X \subseteq V\left(G_{k}\right)$. If $\mathrm{mm}(X)<k$, then one of $X$ and $X^{c}$ is small.

Proof. Suppose that neither $X$ nor $X^{c}$ is small. Then we can choose $i_{1}, i_{2}$ with $1 \leq i_{1}, i_{2} \leq k$ such that $R_{i_{1}} \subseteq X$ and $R_{i_{2}} \subseteq X^{c}$. Now we may choose an edge from each column of $G_{k}$ with endpoints one in $X$ and the other in $X^{c}$. Since these edges form a matching of size $k$, we have $\mathrm{mm}(X) \geq k$, a contradiction.

Lemma 4.4. If $X \subseteq V\left(G_{k}\right)$ is small, then there exist $i, j$ such that $R_{i} \cap X=C_{j} \cap X=\emptyset$.

Proof. Suppose that $\left|R_{i} \cap X\right|>0$ for all $i$. Since $X$ is small, $R_{i} \cap X^{c} \neq \emptyset$. Thus, $G\left[R_{i}\right]$ contains an edge between $X$ and $X^{c}$ for every $i$. These edges show that $\mathrm{mm}(X) \geq k$, a contradiction. Likewise, $C_{j} \cap X=\emptyset$ for some $j$.

Lemma 4.5. If $X_{1} \cup X_{2} \cup X_{3}=V\left(G_{k}\right)$, then one of $X_{1}, X_{2}$, and $X_{3}$ is not small.

Proof. We prove by induction on $k$. The lemma is trivial when $k=2$. Assume that $k>2$ and the lemma is true for $k-1$. To prove by contradiction, let us suppose that all of $X_{1}, X_{2}$, and $X_{3}$ are small. Note that each row or column intersects at least two of $X_{1}, X_{2}$ and $X_{3}$.

Firstly we suppose that $R_{k} \cup C_{k}$ intersects $X_{t}$ for all $t \in\{1,2,3\}$. We consider the $(k-1) \times(k-1)$-grid $G_{k-1}=G_{k} \backslash\left(R_{k} \cup C_{k}\right)$ with sets $X_{t}^{\prime}=X_{t} \backslash\left(R_{k} \cup C_{k}\right)$ for each $t \in\{1,2,3\}$ so that $X_{1}^{\prime} \cup X_{2}^{\prime} \cup X_{3}^{\prime}=V\left(G_{k-1}\right)$. By the induction hypothesis, we may assume that $X_{1}^{\prime}$ is not small in $G_{k-1}$. That is, $\mathrm{mm}_{G_{k-1}}\left(X_{1}^{\prime}\right) \geq k-1$ or $X_{1}^{\prime}$ contains a row of $G_{k-1}$. If $\mathrm{mm}_{G_{k-1}}\left(X_{1}^{\prime}\right) \geq k-1$, then $G_{k-1}$ has a matching of size $k-1$ between $X_{1}^{\prime}$ and $V\left(G_{k-1}\right) \backslash X_{1}^{\prime}$. Since $G_{k}$ has an edge in $G_{k}\left[R_{k} \cup C_{k}\right]$ with one end in $X_{1}$ and the other in $X_{1}^{c}$, we obtain a matching of size $k$ in $G_{k}\left[X_{1}, X_{1}^{c}\right]$ showing that $m m\left(X_{1}\right) \geq k$ and $X_{1}$ is not small. Hence we may assume that $\operatorname{mm}_{G_{k-1}}\left(X_{1}^{\prime}\right)<k-1$ and $X_{1}^{\prime}$ contains a row $R^{\prime}$ of $G_{k-1}$. Since we assumed $X_{1}$ to be small, one of the columns of $G_{k}$ does not intersect $X_{1}$ by Lemma 4.4 but it must be $C_{k}$; all other columns intersect with $R^{\prime}$. On the other hand, by Lemma 4.2, $X_{1}^{\prime}$ also contains a column of $G_{k-1}$ and $R_{k}$ does not intersect $X_{1}$. Thus $\left(R_{k} \cup C_{k}\right) \cap X_{1}=\emptyset$, a contradiction to our assumption that $R_{k} \cup C_{k}$ intersects all of $X_{1}, X_{2}$ and $X_{3}$.

Therefore we may assume that for every choice $i, j \in\{1, k\}, R_{i} \cup C_{j}$ does not intersect all $X_{t}$ at the same time. Since each row and column intersects at least two of $X_{1}, X_{2}$ and $X_{3}$, if $R_{1} \cup R_{k}$ meets all $X_{t}$, then either $R_{1} \cup C_{k}$ or $R_{k} \cup C_{k}$ meets all $X_{t}$ so that we assume each of $R_{1}$ and $R_{k}$ intersects $X_{1}$ and $X_{2}$ but not $X_{3}$. It follows also that each of $C_{1}$ and $C_{k}$ intersects $X_{1}$ and $X_{2}$ but not $X_{3}$.

We shall show $m m\left(X_{1}\right)+m m\left(X_{2}\right) \geq 2 k$ by proving that each column of $G_{k}$ contains either two independent edges from one of $E\left[X_{1}, X_{1}^{c}\right]$ and $E\left[X_{2}, X_{2}^{c}\right]$, or one edge from each set. Those edges form two matchings in $G\left[X_{1}, X_{1}^{c}\right]$ and $G\left[X_{2}, X_{2}^{c}\right]$ respectively whose sizes sum up to at least $2 k$. Thus we get $\mathrm{mm}\left(X_{1}\right) \geq k$ or $\mathrm{mm}\left(X_{2}\right) \geq k$ and one of $X_{1}$ and $X_{2}$ is not small. 
If a column has an edge with one end in $X_{1} \backslash X_{2}$ and the other in $X_{2} \backslash X_{1}$ then we are done. Thus $C_{1}$ and $C_{k}$ are fine. If all columns are as such then we are done. Otherwise, there is a column $C_{i}$ such that $C_{i} \cap X_{2} \subseteq C_{i} \cap X_{1}$. Since $C_{i} \not \subset X_{1}$, we have $\left|C_{i} \cap\left(X_{3} \backslash X_{1}\right)\right|>0$. If $\left|C_{i} \cap\left(X_{3} \backslash X_{1}\right)\right| \geq 2$ then $C_{i}$ has two independent edges in $E\left[X_{1}, X_{1}^{c}\right]$. Thus we assume $\left|C_{i} \cap\left(X_{3} \backslash X_{1}\right)\right|=1$, that is, $\left|C_{i} \cap X_{1}\right|=k-1$. By Lemma 4.4 we choose a column $C_{j}$ not intersecting with $X_{1}$, and between $C_{i}$ and $C_{j}$ we can find $k-1$ independent row-edges in $E\left[X_{1}, X_{1}^{c}\right]$. Since $C_{1}$ and $C_{k}$ are not in this area, we may choose an edge from $G\left[C_{1}\right] \cap G\left[X_{1}, X_{1}^{c}\right]$ and $G\left[X_{1}, X_{1}^{c}\right]$ has a matching of size $k$, showing that $\mathrm{mm}\left(X_{1}\right) \geq k$ and $X_{1}$ is not small. This final contradiction completes the proof.

Lemma 4.6. Let $\mathcal{T}$ be the set of all small subsets of $V\left(G_{k}\right)$. The set $\mathcal{T}$ is a tangle in $G_{k}$ of order $k$.

Proof. The first and second axioms follow from Lemmas 4.3 and 4.5 respectively. For each $x \in V\left(G_{k}\right)$, the set $V\left(G_{k}\right) \backslash\{x\}$ contains a row and thus not in $\mathcal{T}$.

By Theorem 2.5, Lemma 4.6 implies $\operatorname{mmw}\left(G_{k}\right)>k-1$. Since the branchwidth of $G_{k}$ is $k$, by Theorem $4.1, \operatorname{mmw}\left(G_{k}\right)$ is at most $k$.

Theorem 4.7. The $k \times k$-grid has maximum matching width $k$ for $k \geq 2$.

\section{Acknowledgments}

We thank Jan Arne Telle for introducing the first problem. We also thank Sang-il Oum for giving an important idea to compute the maximum matching width of the $k \times k$-grid.

The first author was supported by the National Research Foundation of Korea (NRF) grant funded by the Korea government (MSIT) (No. NRF-2017R1A2B4005020). The second author was partially supported by Basic Science Research Program through the National Research Foundation of Korea (NRF) funded by the Ministry of Education (NRF2016R1D1A1B03932398).

\section{References}

[1] S. Arnborg, A. Proskurowski, D.G. Corneil, Forbidden minors characterization of partial 3-trees, Discrete Math. 80 (1) (1990) 1-19.

[2] H.L. Bodlaender, S. Kratsch, V.J. Kreuzen, O. Joung Kwon, S. Ok, Characterizing width two for variants of treewidth, Discrete Appl. Math. 216 (1) (2017) 29-46.

[3] H.L. Bodlaender, D.M. Thilikos, Graphs with branchwidth at most three, J. Algorithms 32 (2) (1999) 167-194.

[4] B. Courcelle, The monadic second-order logic of graphs. I. Recognizable sets of finite graphs, Inform. and Comput. 85 (1) (1990) $12-75$.

[5] Z. Dvořák, A.C. Giannopoulou, D.M. Thilikos, Forbidden graphs for tree-depth, European J. Combin. 33 (5) (2012) 969-979. EuroComb '09.

[6] F. Gavril, The intersection graphs of subtrees in trees are exactly the chordal graphs, J. Combinatorial Theory Ser. B 16 (1974) $47-56$.

[7] V. Jelínek, The rank-width of the square grid, Discrete Appl. Math. 158 (7) (2010) 841-850.

[8] J. Jeong, S.H. Sther, J.A. Telle, Maximum matching width: New characterizations and a fast algorithm for dominating set, in: T. Husfeldt, I. Kanj (Eds.), 10th International Symposium on Parameterized and Exact Computation, IPEC 2015, in: Leibniz International Proceedings in Informatics (LIPIcs), vol. 43, Schloss Dagstuhl-Leibniz-Zentrum fuer Informatik, Dagstuhl, Germany, 2015, pp. 212-223.

[9] N.G. Kinnersley, M.A. Langston, Obstruction set isolation for the gate matrix layout problem, Discrete Appl. Math. 54 (2) (1994) $169-213$.

[10] D. Lokshtanov, D. Marx, S. Saurabh, Known algorithms on graphs of bounded treewidth are probably optimal, in: Proceedings of the Twenty-SEcond Annual ACM-SIAM Symposium on Discrete Algorithms, SIAM, Philadelphia, PA, 2011, pp. 777-789.

[11] R. Read, R. Wilson, An Atlas of Graphs, Oxford Science Publications, Clarendon Press, 1998.

[12] N. Robertson, P. Seymour, Graph minors. X. Obstructions to tree-decomposition, J. Combin. Theory Ser. B 52 (2) (1991) 153-190.

[13] N. Robertson, P. Seymour, Graph minors. XX. Wagner's conjecture, J. Combin. Theory Ser. B 92 (2) (2004) 325-357. Special Issue Dedicated to Professor W.T. Tutte.

[14] S.H. Sæther, J.A. Telle, Personal communication.

[15] S.H. Sæther, J.A. Telle, Between treewidth and clique-width, Algorithmica 75 (1) (2016) 218-253.

[16] A. Satyanarayana, L. Tung, A characterization of partial 3-trees, Networks 20 (3) (1990) 299-322.

[17] P. Seymour, R. Thomas, Graph searching and a min-max theorem for tree-width, J. Combin. Theory Ser. B 58 (1) (1993) 22-33.

[18] W. Tutte, A theory of 3-connected graphs, Indag. Math. 64 (1961) 441-455.

[19] J.M.M. van Rooij, H.L. Bodlaender, P. Rossmanith, Dynamic programming on tree decompositions using generalised fast subset convolution, in: Algorithms-ESA 2009, in: Lecture Notes in Comput. Sci., vol. 5757, Springer, Berlin, 2009, pp. 566-577.

[20] M. Vatshelle, New Width Parameters of Graphs (Ph.D. thesis), University of Bergen, 2012.

[21] J.A. Wald, C.J. Colbourn, Steiner trees, partial 2-trees, and minimum IFI networks, Networks 13 (2) (1983) 159-167. 